\title{
Filtering False Detections of Small Multiple Sclerosis Lesions using Fuzzy Regional Analysis
}

\author{
F. X. Aymerich, Member, IEEE, P. Sobrevilla, Senior Member, IEEE, E. Montseny, Member, IEEE, \\ and A. Rovira
}

\begin{abstract}
This paper introduces a method to filter false detections of small multiple sclerosis lesions in magnetic resonance images based on the analysis of regional features. The proposed method considers as starting point the results of an earlier work in which, through the use of fuzzy rules, the image pixels showing hyperintensity were detected. The regional analysis of the results obtained at previous work allows extracting some features with differentiation capability between small multiple sclerosis lesions and false detections. These features are introduced as restrictions for obtaining a new and improved fuzzy membership function associated with the presence of hyperintensity in these images. Results show an important reduction of the number of false detections preserving the small multiple sclerosis lesions previously detected.
\end{abstract}

\section{INTRODUCTION}

$\mathrm{M}$ ULTIPLE sclerosis (MS) is a disease of the central nervous system characterized by the destruction of the myelin that encases the axons. This demyelinizating process results in an inhibition of neural transmission and causes the appearance of several clinical symptoms, such as motor and sensory disturbances, paralysis or visual alterations [1]. These areas of demyelinization, or lesions, can be observed by magnetic resonance imaging (MRI).

MRI is the most sensitive technique for visualizing MS lesions. In an MRI examination, MS lesions can be visualized using different sequences. Among all them, sequences with dual acquisition of proton density (PD-) and T2-weighted images are the most commonly used to evaluate lesion volume in MS [2]. In clinical trials on MS including MRI, the evaluation of lesion volume on PD- and T2weighted images is one of the measures that use to be

This work has been partially supported by the Spanish CICYT Project TIN2007-68063

F. X. Aymerich is with the Magnetic Resonance Unit - IDI, Vall Hebron University Hospital, E-08035 Barcelona, Spain (phone: +34934286034; fax: +34934286059; e-mail: xavier.aymerich@idi-cat.org) and with the Computational Engineering and Industrial Systems Department, Technical University of Catalonia, E-08034 Barcelona, Spain (e-mail: xavier.aymerich@upc.edu).

P. Sobrevilla is with the Applied Mathematics II Department, Technical University of Catalonia, E-08034 Barcelona, Spain (e-mail: pilar.sobrevilla@upc.edu).

E. Montseny is with the Computational Engineering and Industrial Systems Department, Technical University of Catalonia, E-08034 Barcelona, Spain (e-mail: eduard.montseny@upc.edu).

A. Rovira is with the Magnetic Resonance Unit - IDI, Vall Hebron University Hospital, E-08035 Barcelona, Spain (e-mail: alex.rovira@idicat.org) considered [3],[4].

However, the detection of regions corresponding to lesion in magnetic resonance images is an arduous and complex task. This is due to the fact that detection of lesions involves the analysis of several images with different anatomical features, and presents a high dependence of the contrast-tonoise ratio in the images. Moreover, MS lesions are inherently fuzzy with an uncertainty component mainly in the definition of their boundaries [3]. Therefore, the introduction of an automatic algorithm for detecting MS lesions would help to reduce the inter- and intra-observer variability in the evaluation of lesion volumes.

The difficulty to avoid false detections is one of the main problems to achieve a full automatic detection of MS lesions. Most of false detections are related to the detection of small regions in the images and the difficulty to discriminate them from small MS lesions. In this way, although some authors, [5]-[9], have developed algorithms for detecting MS lesions, these algorithms require removing false detections manually or that the detection excludes detected regions below a minimum size.

This problem is related to the complexity of MRI images and the difficulty for an automatic algorithm to recognize the lesions as a human expert, although automatic algorithms have advantages such as capability of a better delineation and faster processing [3]. Thus, the reduction of false detections is essential to minimize the operator assistance using an algorithm.

Much of the complexity to detect MS lesions is due to the detection of their boundary pixels. The case of small lesions, the greater proportion of boundary pixels in relation to lesions of greater size is an additional difficulty to the characteristic noise or non-homogeneities of magnetic resonance images.

In this paper we present an algorithm to filter false detections in magnetic resonance images. Specifically we focused on the differentiation between small MS lesions and false detections obtained using our previous fuzzy detection algorithm [10]. With this aim will be fed into the algorithm regional characteristics of the detections that were not considered at previous work. The algorithm presented in this paper is part of an ongoing work in the research field that we have been developing during last years.

The paper is structured as follows: Section II describes the characteristics of data used as starting point to this work. 
Next section gives an overview of the regional analysis included in the proposed algorithm. At section four we present some results and last section is devoted to presenting the conclusions of the proposed work.

\section{PROCEDURE APPLIED TO OBTAIN THE WORKING IMAGE DATASET}

We considered eight proton density and eight T2-weighted brain axial images acquired in a 1.5T Magnetom Vision MR System (Siemens, Erlangen, Germany) using a dual turbo spin-echo sequence (TR/ TE/ NEX/ Matrix/ FOV/ Thickness: 3000ms/ 12-80ms/ 1/ 256x256/ 250mm/3mm). These images corresponded to axial slices associated with four representative brain locations. Half of these images belonged to a MS patient with high lesion load, whereas the other half corresponded to a low lesion load MS patient.

We applied the fuzzy detection algorithm described in [10] to detect small MS lesions. This algorithm took into account the following definitions:

\section{Definition 2.1}

A pixel, $p_{i j}$, presents hyperintensity if its gray level, $g\left(p_{i j}\right)$ in PD- and T2-weighted images is high enough in relation to its near surrounding.

\section{Definition 2.2}

A region, $R_{k}$, is a small MS lesion if:

i.- $R_{k}$ is located within the encephalic parenchyma,

ii.- $\forall \mathrm{p}_{\mathrm{ij}} \in \mathrm{R}_{\mathrm{k}}, \mathrm{p}_{\mathrm{ij}}$ presents hyperintensity, and

iii.- $R_{k}$ is contained within a $5 \times 5$ raster window with a minimum size of 3 pixels.

For implementing these definitions we considered, in a first step, a detection procedure based on the analysis of hyperintensity in both PD- and T2-weighted images, which involved gray level analysis and radial analysis of gray-level differences within $7 \times 7$ raster windows. Afterwards, for each pixel $p_{i j}$ we obtained its membership degree to the fuzzy set $F_{H}$ associated with the presence of hyperintensity in both images. This way, for each slice we obtained the image $I_{H}$, for which at each position $(i, j) I_{H}(i, j)$ provides the membership degree of the pixel to the fuzzy set $F_{H}$, i.e. $I_{H}(i, j)=\mu_{H}\left(p_{i j}\right)$. Then, these images were deffuzzified considering an $\alpha$-cut with $\alpha=0.5$, and applying two filters: a first that constrained the size of detections within a $5 \times 5$ raster window with a minimum of 3 pixels; and a second that removed all detections located on cerebrospinal fluid locations.

Fig. 1 shows an example of the original images, (a) and (b), and the pixels detected, (c). In these images we can observe that, although most of the pixels corresponding to small MS lesions (brighter pixels surrounded by circles in (c)) were detected, there are too many false positives (gray pixels in image (c)) that size and location filters failed to eliminate.

At the final step of the algorithm, for each slice we get information on: the image $I_{H}(i, j)$ that provides the membership degree of the pixels to the fuzzy set $F_{H}$ after detection and filtering steps; its gray level in PD- and T2weighted images; the image containing a binary mask of small MS lesions, which was obtained by manual tracing on these images using Dispimage software [11]; and image $I_{\alpha}$ obtained after the deffuzification process.

Both original images and all the work described in this section are the starting point considered at next section. To simplify reference to this process, which is applied to obtain the working image dataset, from now on we will refer to it as initial algorithm.

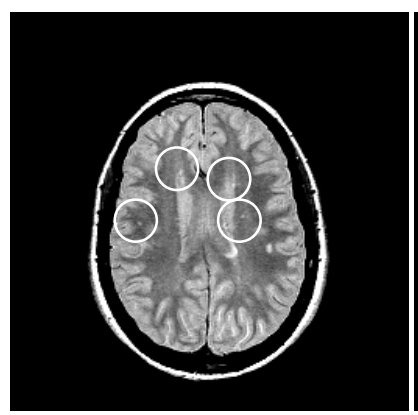

(a)

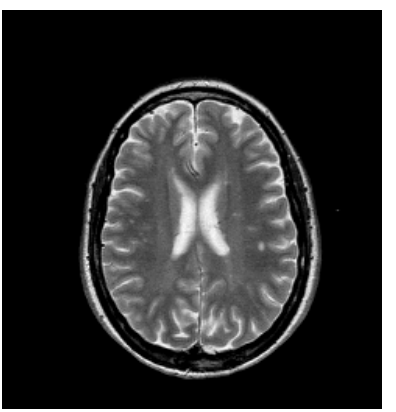

(b)

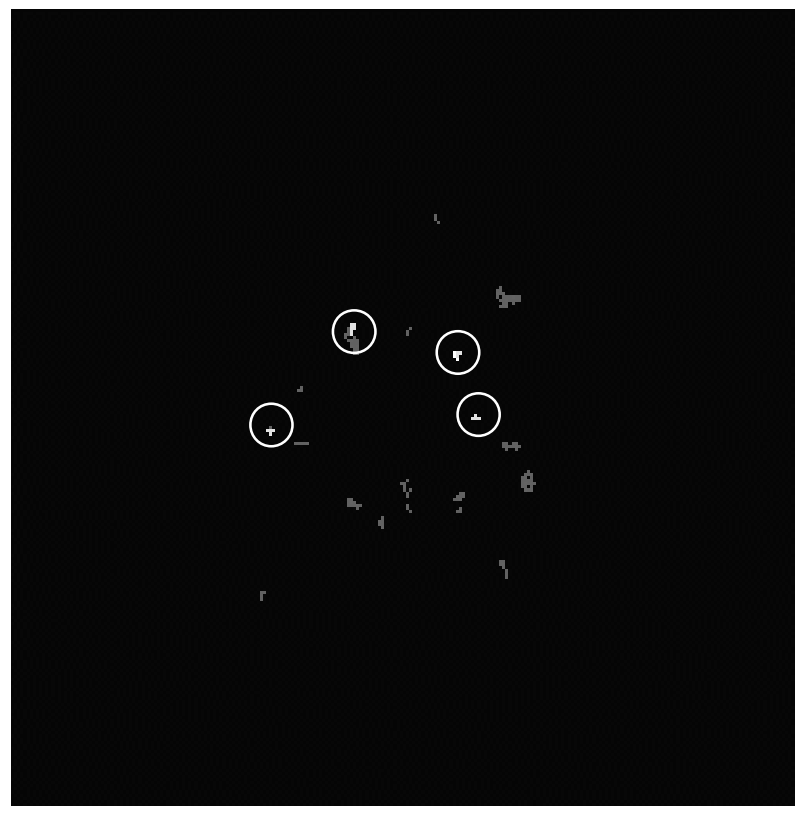

(c)

Fig. 1. Example of initial detection. (a) PD-weighted image. (b) T2-weighted image. (c) Detection resulting of initial detection where brighter pixels surrounded by circles correspond to detected small MS lesions, whereas medium-dark gray regions correspond to false detections.

\section{PROPOSED ALGORITHM DESCRIPTION}

The aim of the algorithm here presented is to filter out the false positives obtained in the initial detection algorithm. To 
carry out this aim we will take into consideration the regional features corresponding to the appearance of small MS lesions in the images. These features will be obtained from the analysis of the regions detected.

Next sections describe the main steps of the proposed algorithm, than can be summarized as follows: $A$. Description of regional features considered to filter out false detections; $B$. Process followed for getting the improved hyperintensity membership function; and $C$. Defuzzification process and quality measures considered for evaluating the obtained results.

\section{A. Regional features}

To implement the algorithm we have introduced regional features of small MS lesions based on the analysis of their characteristics.

In order to filter out false detections of MS lesions we have considered seven features based on: 1) hyperintensity membership degrees of the pixels, $\mu_{\mathrm{H}}\left(p_{i j}\right)$;2) gray level of the pixels within detected regions; and 3) size of detected regions.

Then, for each region $R_{n} 1 \leq n \leq N$ (white pixels in Fig. 2), detected at the initial algorithm such that $\#\left(R_{n}\right)=M_{n}$, we defined the following regional features:

1) Features based on the presence of hyperintensity: Let $\mu_{H}^{R_{n}}$, be the hyperintensity membership degree associated to the pixels $p_{i j} \in R_{n}$. Then, the feature associated with the hyperintensity of the region $R_{n}$, is given by:

\section{Definition 3.1}

The hyperintensity feature associated with region $R_{n}$, $M H_{R_{n}}$, is given by the mean value of the hyperintensity membership degrees of the pixels in $R_{n}$, i.e.:

$$
M H_{R_{n}}=\frac{1}{M_{n}} \sum_{p_{i j} \in R_{n}} \mu_{H}^{R_{n}}
$$

2) Features based on the gray level: Let $I_{P D}(\mathrm{i}, \mathrm{j})$ and $I_{T 2}(\mathrm{i}, \mathrm{j})$ be the gray levels of pixel $p_{i j}$ at the PD- and T2weighted images respectively. Then, we define the image $I_{P D T 2}$ as the geometric mean of $I_{P D}$ and $I_{T 2}$ i.e., if $(i, j)$ is the location of pixel $p_{i j}, I_{P D T 2}(\mathrm{i}, \mathrm{j})$ is given by:

$$
I_{P D T 2}(i, j)=\sqrt{I_{P D}(i, j) I_{T 2}(i, j)}
$$

Based on this definition we consider the images, $I_{P D T 2}^{R_{n}}$ and $I_{P D T 2}^{P R_{n}}$, such that

$$
I_{P D T 2}^{R_{n}}(i, j)= \begin{cases}I_{P D T 2}(i, j) & \text { if } p_{i j} \in R_{n} \\ 0 & \text { otherwise }\end{cases}
$$

$$
I_{P D T 2}^{P R_{n}}(i, j)= \begin{cases}I_{P D T 2}(i, j) & \text { if } p_{i j} \in P R_{n} \\ 0 & \text { otherwise }\end{cases}
$$

where $P R_{n}$ is the one pixel width outer edge of the region $R_{n}$ (light gray pixels in Fig. 2), and $\#\left(P R_{n}\right)=P_{n}$.

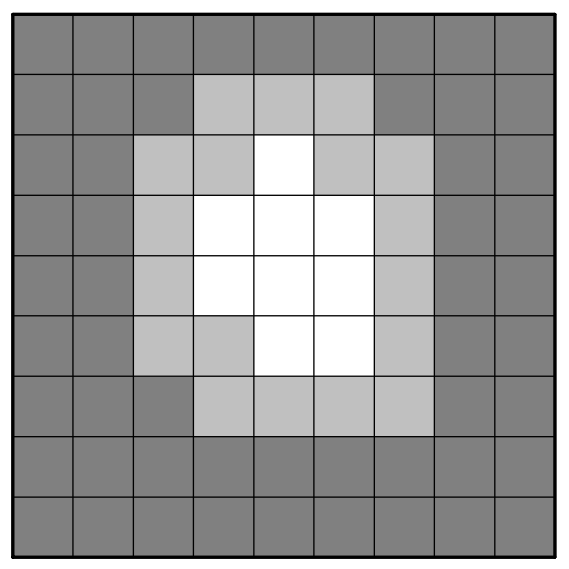

Fig. 2. Example of the pixels considered in the definition of $R_{n}$ (white) and $P_{R}$.(light gray). As it can be appreciated, $P_{n}$ is constituted by the one pixel width edge of $R_{n}$, and the dark gray region is the environment of $R_{n}$ that has not been considered for analysis purposes.

Then, the features associated with the intensity gray levels of pixels in region $R_{n}$ are: $M P_{P R_{n}}$, the mean gray level of pixels in $I_{P D T 2}^{P R_{n}} ; S D P_{P R_{n}}$, the standard deviation of the gray level of pixels in $I_{P D T 2}^{P R_{n}} ; G L R_{R_{n}}$, the quotient between the mean gray levels in $I_{P D T 2}^{P R_{n}}$ and $I_{P D T 2}^{R_{n}}$; and $C V R_{R_{n}}$, the covariance of the gray levels in $I_{P D T 2}^{R_{n}}$. These features are given by the following definitions:

Definition 3.2

$M P_{P R_{n}}$ is the feature associated with the mean gray level in $I_{P D T 2}^{P R_{n}}$, i.e.:

$$
M P_{P R_{n}}=\frac{1}{P_{n}} \sum_{p_{i j} \in P R_{n}} I_{P D T 2}^{P R_{n}}\left(p_{i j}\right)
$$

Definition 3.3

$S D P_{P R_{n}}$ is the feature associated with the standard deviation of gray level in $I_{P D T 2}^{P R_{n}}$, i.e.:

$$
S D P_{P R_{n}}=\sqrt{\frac{\sum_{p_{i j} \in P R_{n}}\left(I_{P D T 2}^{P R_{n}}\left(p_{i j}\right)-\frac{1}{P_{n}} \sum_{p_{i j} \in P R_{n}} I_{P D T 2}^{P R_{n}}\left(p_{i j}\right)\right)}{P_{n}-1}}
$$




\section{Definition 3.4}

$G L R_{R_{n}}$ is the feature associated with the quotient between mean gray levels of $I_{P D T 2}^{P R_{n}}$ and $I_{P D T 2}^{R_{n}}$, i.e.:

$$
G L R_{R_{n}}=\frac{\frac{1}{P_{n}} \sum_{p_{i j} \in P R_{n}} I_{P D T 2}^{P R_{n}}\left(p_{i j}\right)}{\frac{1}{M_{n}} \sum_{p_{i j} \in R_{n}} I_{P D T 2}^{R_{n}}\left(p_{i j}\right)}
$$

\section{Definition 3.5}

$C V R_{R_{n}}$ is the feature associated with the quotient between the standard deviation and the mean of gray levels in $I_{P D T 2}^{R_{n}}$, i.e.:

$$
C V R_{R_{n}}=\frac{\sqrt{\frac{\sum_{p_{i j} \in R_{n}}\left(I_{P D T 2}^{R_{n}}\left(p_{i j}\right)-\frac{1}{M_{n}} \sum_{p_{i j} \in R_{n}} I_{P D T 2}^{R_{n}}\left(p_{i j}\right)\right)}{M_{n}-1}}}{\frac{1}{M_{n}} \sum_{p_{i j} \in R_{n}} I_{P D T 2}^{R_{n}}\left(p_{i j}\right)}
$$

3) Features based on region size: Let $p_{i j}$ and $p_{k l}$ be pixels belonging to $R_{n}$. Then, distances between these pixels in the $x$ and $y$ directions, $d_{x}\left(p_{i j}, p_{k l}\right)$ and $d_{y}\left(p_{i j}, p_{k l}\right)$, are given by:

$$
\begin{aligned}
& d_{x}\left(p_{i j}, p_{k l}\right)=|i-k| \\
& d_{y}\left(p_{i j}, p_{k l}\right)=|j-l|
\end{aligned}
$$

Using these distances we define two features associated to region size: $D X Y_{R_{n}}$, the quotient between maximum distances in $x$ and $y$ directions; and $F F_{R_{n}}$, the filling factor of $R_{n}$. These features are given by the following definitions:

\section{Definition 3.6}

$D X Y_{R_{n}}$ is the feature associated with the quotient between

$$
\begin{gathered}
\max _{p_{i j}, p_{k l} \in R_{n}}\left(d_{x}\left(p_{i j}, p_{k l}\right)\right) \text { and } \max _{p_{i j}, p_{k l} \in R_{n}}\left(d_{y}\left(p_{i j}, p_{k l}\right)\right) \text {, i.e.: } \\
D X Y_{R_{n}}=\frac{\max _{p_{i j}, p_{k l} \in R_{n}}\left(d_{x}\left(p_{i j}, p_{k l}\right)\right)}{\max _{p_{i j}, p_{k l} \in R_{n}}\left(d_{y}\left(p_{i j}, p_{k l}\right)\right)}
\end{gathered}
$$

Definition 3.7

The filling factor, $F F_{R_{n}}$, is the feature associated with the quotient between $M_{n}$ and the product of the maximum, in $R_{n}$, of the distances in the $\mathrm{x}$ and $\mathrm{y}$ directions, i.e.:

$$
F F_{R_{n}}=\frac{M_{n}}{\max _{p_{i j}, p_{k l} \in R_{n}}\left(d_{x}\left(p_{i j}, p_{k l}\right)\right)_{p_{i j}, p_{k l} \in R_{n}}\left(d_{y}\left(p_{i j}, p_{k l}\right)\right)}
$$

\section{B. Features evaluation}

Features defined at previous section were evaluated for all the regions $R_{n}$ detected in the eight considered slices. This evaluation was done separately for correct and false detections. Table I shows minimum and maximum values obtained for the seven features (first column) in case of small lesions (columns two and three) and for false detections (columns four and five).

Data in this table show that most of the features do not share the same intervals for small MS lesions and false detections.

If we focus on each group of features, it can be appreciated that:

1) Features based on the presence of hyperintensity: The feature $M H_{R_{n}}$ (row three), whose values are in the interval $[0,1]$, shows greater values for small MS lesions than for false detections, due to small MS lesions match better to the concept of hyperintensity than other regions in the images.

2) Features based on the gray level: Values of the features $M P_{P R_{n}}$ and $S D P_{P R_{n}}$ (rows four and five) show differences in both extremes (Min and Max), although these differences are higher on the right side of the interval (Max), what is due to the gray-level homogeneity in the outer periphery is lower for false detections than for MS lesions. The feature that considers the relation between gray levels within a region $R_{n}$ and its periphery $P R_{n}, G L R_{R_{n}}$ (row six), also shows a lower range of values for small MS lesions. This is basically due to the higher variability on the outer periphery for false detections. As $C V R_{R_{n}}$ (row seven) showed similar interval of values for both intervals, differences in $G L R_{R_{n}}$ are due mainly to the variability in $P R_{n}$.

3) Features based on region size: Features $D X Y_{R_{n}}$ and $F F_{R_{n}}$ (rows seven and eight) show that false detections presented a greater difference between $x$ and $y$ dimensions, as can be inferred from both extremes (Min and Max) of $D X Y_{R_{n}}$ and the lower minimum value of $F F_{R_{n}}$.

TABLE I

EVALUATION OF FEATURES

\begin{tabular}{llclc}
\hline \hline \multirow{2}{*}{ Features } & \multicolumn{2}{c}{ Small MS lesions } & \multicolumn{2}{c}{ False detections } \\
\cline { 2 - 5 } & Min & Max & Min & Max \\
\hline$M H_{R_{n}}$ & 0.63 & 0.94 & 0.53 & 0.87 \\
$M P_{P R_{n}}$ & 2.56 & 54.71 & 4.67 & 183.38 \\
$S D P_{P R_{n}}$ & 3.61 & 24.97 & 5.01 & 78.33 \\
$G L R_{R_{n}}$ & 0.07 & 0.56 & 0.11 & 1.07 \\
$C V R_{R_{n}}$ & 0.03 & 0.63 & 0.04 & 0.61 \\
$D X Y_{R_{n}}$ & 0.50 & 1.50 & 0.25 & 5.00 \\
$F F_{R_{n}}$ & 0.50 & 1.00 & 0.28 & 1.00 \\
\hline \hline
\end{tabular}

Min: Minimum, Max: Maximum 
Previous considerations about features show as most of them presented differences by themselves between small lesions and false detections. However, as we have three groups of features, those associated with hyperintensity, gray level and region size, we also studied their separability considering pairs of features of different groups.

So, we studied the features individually and paired because we had not previous knowledge about the dependence among them, and we wanted to filter so many false detections as possible.

Both in the individual and paired analysis of the features, we used scatter plots to visualize the distribution of detections corresponding to small MS lesions and false detections. We discriminated between them selecting straight lines that divided the region including only false detections from those that included both types of detections. Then, we repeated this graphical process for all the features, selecting those restrictions that allowed a greater discrimination between false detections and small MS lesions.

Fig. 3 shows an example of an scatter plot in which we have plotted a gray-level feature, $C V R_{R_{n}}$, and a feature based on the size of the detected region, $F F_{R_{n}}$. In this plot, we have marked small MS lesions and false detections with square markers and hollow circle markers, respectively. A solid line discriminates between regions with (above this line) and without (below this line) small MS lesion detections. This solid line was obtained by analysis of the distribution of detections on the plot according to features $C V R_{R_{n}}$ and $F F_{R_{n}}$, selecting a straight line that discriminate between the region without detections corresponding to small MS lesion and the region that include both detections. Then, we used the equation of this straight line for defining a restriction that separated both regions.

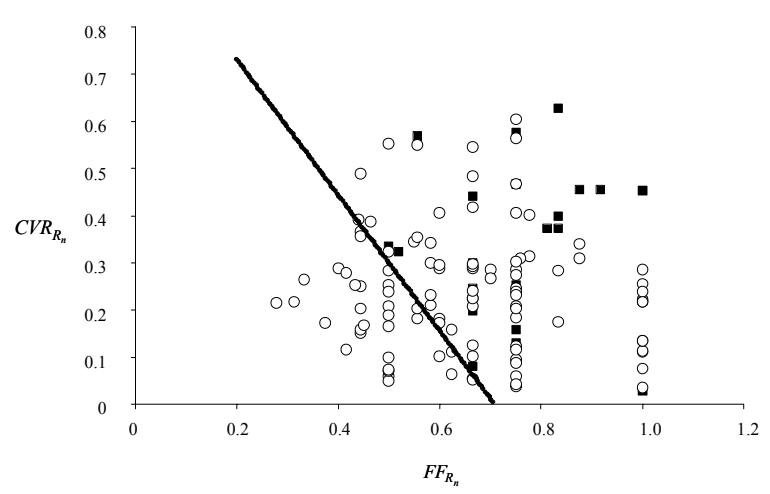

Fig. 3. Scattered Plot of $C V R_{R_{n}}$ vs. $F F_{R_{n}}$. Small MS lesions are represented by square markers, whereas hollow circle markers correspond to false detections. A solid straight line divides regions without (below this line) and with (above this line) small MS lesions.
Following this procedure we obtained the next restrictions:

$C_{1}^{R_{n}}= \begin{cases}1 & \text { if } G L R_{R_{n}}>0.125 \frac{M H_{R_{n}}-0.65}{0.049}+0.445 \\ 0 & \text { otherwise }\end{cases}$

$$
\begin{aligned}
& C_{2}^{R_{n}}=\left\{\begin{array}{l}
1 \quad \text { if } C V R_{R_{n}}<-0.24 \frac{F F_{R_{n}}-0.5}{0.167}+0.3 \\
0 \quad \text { otherwise }
\end{array}\right. \\
& C_{3}^{R_{n}}= \begin{cases}1 & \text { if } S D P_{P R_{n}}>27.5 \\
0 & \text { otherwise }\end{cases} \\
& C_{4}^{R_{n}}= \begin{cases}1 & \text { if } M P_{P R_{n}}>60 \\
0 & \text { otherwise }\end{cases} \\
& C_{5}^{R_{n}}= \begin{cases}1 & \text { if } D X Y_{R_{n}}<0.45 \\
0 & \text { otherwise }\end{cases} \\
& C_{6}^{R_{n}}= \begin{cases}1 & \text { if } D X Y_{R_{n}}>1.65 \\
0 & \text { otherwise }\end{cases} \\
& C_{7}^{R_{n}}=\left\{\begin{array}{l}
1 \text { if } F F_{R_{n}}<0.5 \\
0 \text { otherwise }
\end{array}\right. \\
& C_{8}^{R_{n}}=\left\{\begin{array}{l}
1 \text { if } M H_{R_{n}}<0.6 \\
0 \quad \text { otherwise }
\end{array}\right.
\end{aligned}
$$

\section{Obtaining the improved hyperintensity membership function}

The restrictions introduced at previous section were considered for defining the improved hyperintensity membership function $\eta_{H}$. To do it, we took into consideration that only regions corresponding to false detections showed values greater than zero for any of the previous restrictions. Then, the membership degree of the pixels in these regions was modified to its complement. Thus, the improved membership function was given by:

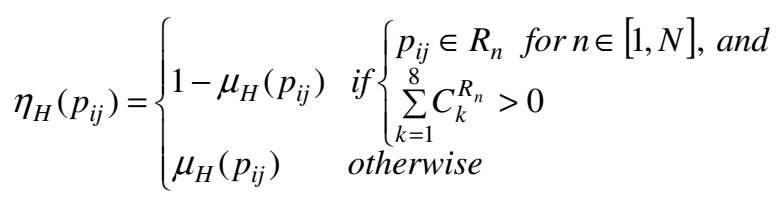

\section{Defuzzification and quality measures}

The improved membership function, $\eta_{H}$, allowed us to obtain for each slice an image $I_{H}^{\prime}$ given by $I_{H}^{\prime}(i, j)=\eta\left(p_{i j}\right)$. For getting the binary images resulting 
from the previous filtering process, we applied the same defuzzification process than in the initial detection algorithm, i.e., we applied the $\alpha$-cut with $\alpha=0.5$ to obtain the crisp representation of detections associated with the presence of hyperintensity.

These binary images were considered to evaluate the quality in the correspondence of these obtained detections in each image to the information provided by the lesion mask. The evaluation was based on some quality indexes that gave information about the efficiency and reliability in the detection.

In the definition of these indexes we considered: the number of detected regions corresponding to small MS lesions, CDR; the number of non-detected regions associated with MS lesions, NDR; and the number of regions corresponding to false detections, FDR. Then, the quality indexes are given by next definitions.

\section{Definition 3.8}

Recall detecting small MS lesions, RSL, is the probability that a true small MS lesion is detected, i.e.:

$$
R S L=\frac{C D R}{C D R+N D R}
$$

This index penalized the small MS regions not detected.

\section{Definition 3.9}

The efficiency in the detection of small MS lesions, EDSL, is the quotient between the number of detected regions corresponding to small MS lesions, and the total number of small MS lesions plus the number of false detections, i.e.:

$$
E D S L=\frac{C D R}{C D R+N D R+F D R}
$$

This index besides penalizing small MS regions not detected, it also penalized the presence of false detections.

\section{Definition 3.10}

Precision in the detection of small MS lesions, PSL, is the probability that a region detected as a small MS lesion by the algorithm is a true detection, i.e.:

$$
P S L=\frac{C D R}{C D R+F D R}
$$

This index only penalized the presence of false detections. These quality indexes were evaluated in the binary images $I_{H}^{\prime}$ corresponding to the eight considered slices.

\section{RESULTS}

The proposed algorithm processed the information provided by the initial detection to obtain the binary images $I_{H}^{\prime}$ according to the procedure described in section III. As a result, we obtained the improvements in the reduction of false detections showed in Table II, and Fig. 4 and 5.

Table II shows the mean values per slice for the number of regions corresponding to false detections, FDR (column five), and the quality indexes RSL, EDSL and PSL (columns two to four). Rows two and three of this table depict the results obtained using the initial and improved detection algorithms, respectively. Looking at column two, it can be appreciated that the index RSL kept invariant, so the detection of small MS lesions was preserved by the proposed algorithm. The values of the efficiency index EDSL and index PSL (columns three and four) increased, although the presence of only a few false detections in some images did not allow to get greater values. It must be emphasized that about a half of false detections were partial detections of greater size lesions, so the number of false detections would be reduced to 2 or 3 per image on average, a more affordable number in case of require the intervention of an operator to remove them.

TABLE II

EVALUATION OF QUALITY IN THE RESULTS

\begin{tabular}{ccccc}
\hline \hline Analysis & $R S L$ & EDSL & PSL & FDR \\
\hline $\begin{array}{c}\text { Initial } \\
\text { detection } \\
\begin{array}{l}\text { Improved } \\
\text { algorithm }\end{array}\end{array}$ & 0.9230 & 0.1506 & 0.1386 & 17.62 \\
\hline \hline
\end{tabular}

RSL: Recall detecting small MS lesions; EDSL: Efficiency in the detection of small MS lesions; PSL: Precision in the detection of small MS lesions; FDR: Number of regions corresponding to false detections.

Fig. 4 shows an example of the results achieved for an image containing small lesions. Image (c) depicts the initial detections for PD- and T2-weighted images, (a) and (b) respectively. The image corresponding to the final detection, (d), shows the results obtained after the application of the improvements given by the proposed algorithm.

It must be emphasized that this image presents an important reduction of false detections (pixels in mediumdark gray level within (d)), from sixteen to four, preserving the correct detections, brighter pixels surrounded by circles in (c) and (d), detected initially.

Fig. 5 shows an example of the results obtained for a slice without small MS lesions. As in the case of Fig. 2, images (a) and (b) show depict the initial detections for PD- and T2weighted images, and in (c) we show the initial detection obtained using the initial algorithm.

Having a look at image (c) it can be observed that appear several false detections distributed everywhere within the 
encephalic parenchyma. However, after applying the proposed only one of the previous false detections remains (pixels in image (d) surrounded by a square around them).

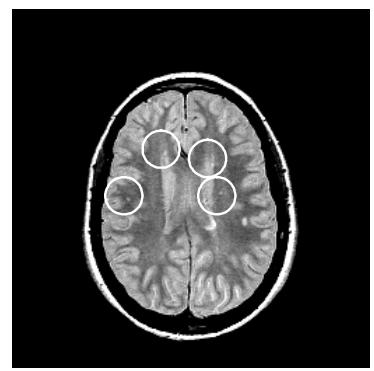

(a)

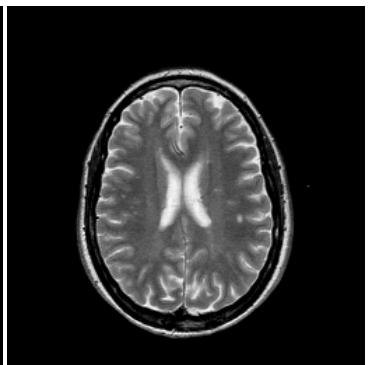

(b)

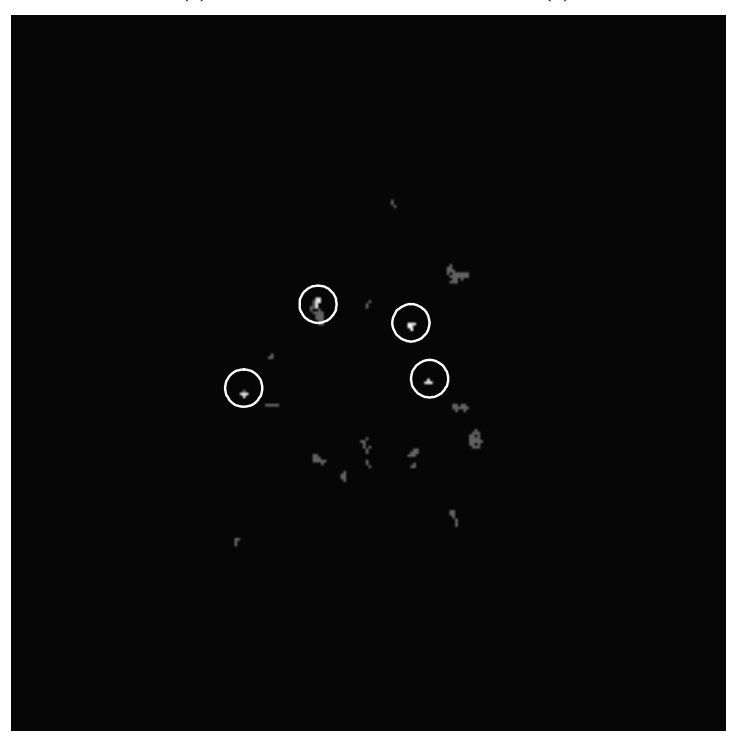

(c)

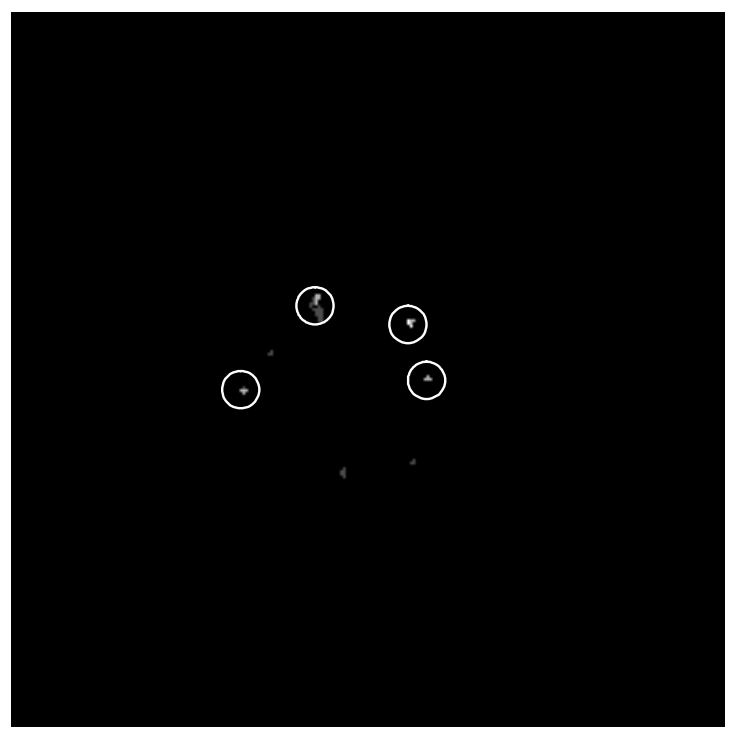

(d)

Fig. 4. Example of the detection obtained for a slice containing small MS lesions. (a) PD-weighted image. (b) T2-weighted image. (c) Image obtained as result of the initial detection. (d) Images obtained after the application of the proposed algorithm. Brighter pixels surrounded by circles point out the detected small MS lesions, whereas medium-dark gray level regions correspond to false detections

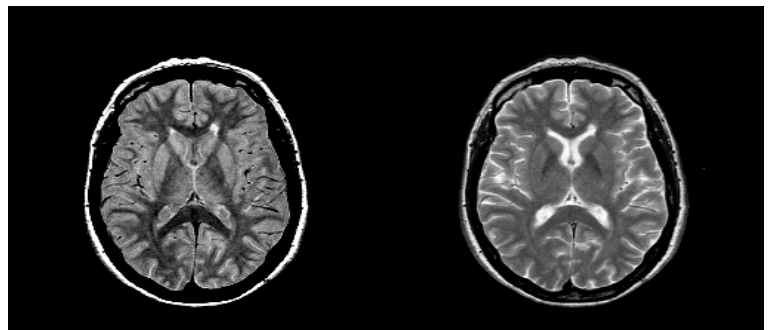

(a)

(b)

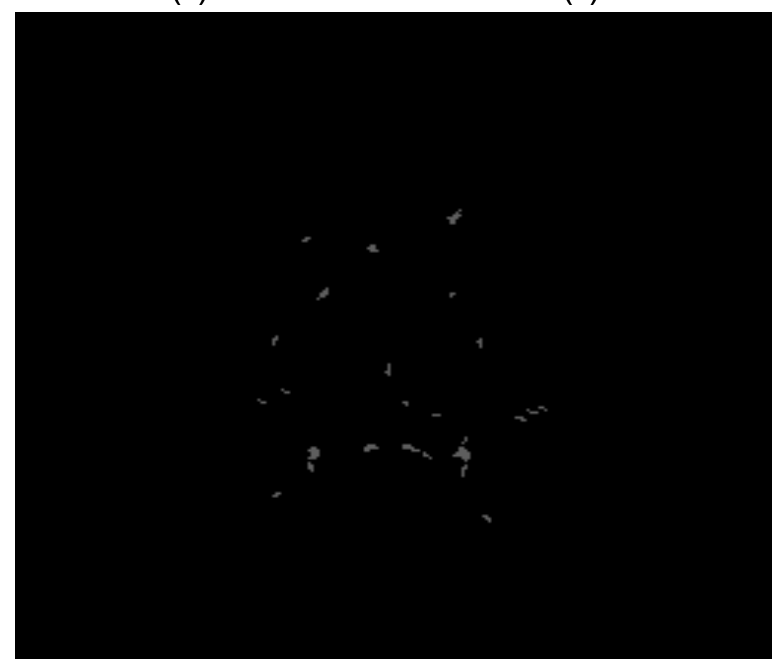

(c)

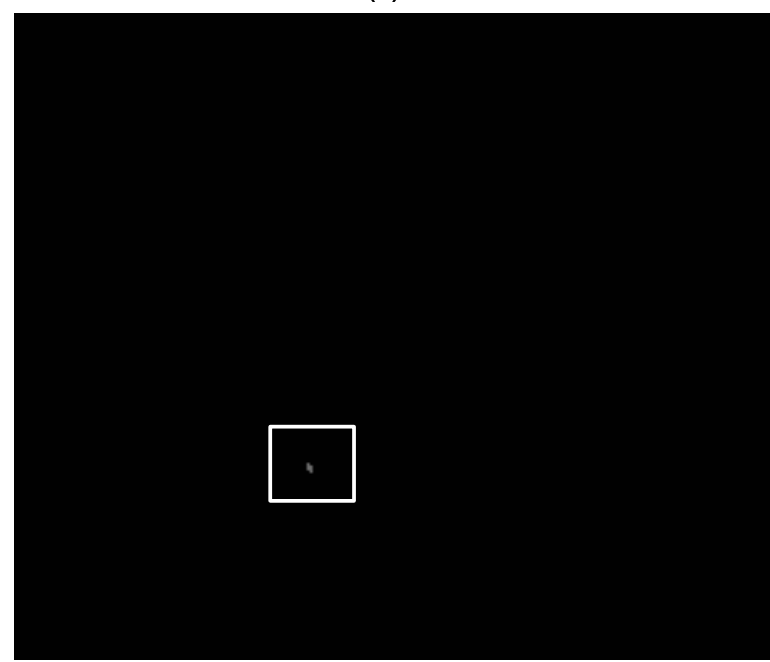

(d)

Fig. 5. Example of the detection obtained for a slice without small MS lesions. (a) PD-weighted image. (b) T2-weighted image. (c) Image obtained as result of the initial detection. (d) Image obtained after the application of the proposed algorithm. Medium-dark graylevel regions correspond to false detections, and region within square is the only false detecting remaining for each slice after the application of the improved algorithm.

\section{CONCLUSIONS}

As conclusion, in this paper we have presented an algorithm to filter false detections of small multiple sclerosis lesions in magnetic resonance images based on the analysis of regional features. We have considered an initial detection obtained from our previous fuzzy detection algorithm [10]. 
As shown by the results presented at previous section, the inclusion of regional constraints has allowed us to obtain a new membership function that improved the previous characterization related to the presence of hyperintensity in PD- and T2-weighted magnetic resonance images.

The algorithm has achieved to obtain images with a much reduced number of false detections without worsening the capability of detecting small MS lesions.

The obtained results suggest the possibility of helping to an existing algorithm to extend it in order to include smaller MS lesions. Moreover, the proposed algorithm can be used as a starting point for the design of an algorithm that detects lesions of greater size. However, the algorithm should be tested with a higher number of images in order to increase its robustness. We plan to carry it out in a future work.

\section{REFERENCES}

[1] R. R. Edelman and Hesselink J R. Clinical magnetic resonance imaging. . W.B. Saunders Company, 1990.

[2] P. A. Narayana, M. Mehta and J. Wolinsky, "Magnetic resonance in multiple sclerosis." in Recent advances in MR imaging and spectroscopy, N. R. Jagannathan, Ed., Jaypee Brothers Medical Publishers Ltd. New Delhi, 2005, pp. 154-185.

[3] D. H. Miller, R. I. Grossman, S. C. Reingold and H. F. McFarland, "The role of magnetic resonance techniques in understanding and managing multiple sclerosis", Brain, vol. 121, no. 1, pp. 3-24, Jan. 1998.
[4] D. H. Miller, M. Filippi, F. Fazekas, J. L. Frediksen, P.M. Matthews P. M. et al., "The role of magnetic resonance imaging within diagnostic criteria for multiple sclerosis", Ann. Neurol., vol. 56, no. 2, pp. 273-278, Aug 2004

[5] J. K. Udupa, L. Wei, S. Samarasekera, Y. Miki, M. A. van Buchem et al., "Multiple sclerosis lesion quantification using fuzzyconnectedness principles", IEEE Trans Med Imaging, vol. 16, no. 5, pp. 598-609, Oct. 1997.

[6] K. Van Leemput, F. Maes, D. Vandermeulen, A. Colchester and P. Suetens, "Automated segmentation of multiple sclerosis lesions by model outlier detection", IEEE Trans Med Imaging, vol. 20, no. 8, pp. 677-688, Aug. 2001.

[7] F. Admiraal-Behloul, D. M. J. van den Heuvel, H. Olofsen, M. J. P. van Osch, J. van der Grond et al., "Fully automatic segmentation of white matter hyperintensities in MR images of the elderly", Neuroimage, vol. 28, no. 3, pp. 607-617, Nov. 2005.

[8] B. R. Sajja, S. Datta, R. He, M. Mehta, R.K. Gupta et al., "Unified Approach for multiple sclerosis lesions segmentation on brain MRI", Ann. Biomed. Eng., vol.34, no. 1, pp. 142-151, Jan 2006.

[9] A. Akselrod-Ballin, M. Galun, J.M. Gomori, M. Filippi, P. Valsasina, et al., "Automatic segmentation and classification of multiple sclerosis in multichannel MRI", IEEE Trans. on Biomedical Engineering, vol. 56, no. 10, pp. 2461-2469, Oct. 2009

[10] F. X. Aymerich, P. Sobrevilla, J. Capellades, E. Grivé, J. Gili et al., "Fuzzy detection of multiple sclerosis small lesions", in Proceedings of the fifth conference of the European Society for Engineering and Medicine (ESEM), pp. 197-198, 1999.

[11] D. L. Plummer, "Dispimage: a display and analysis tool for medical images", Riv. Neuroradiol, vol. 19, pp. 1715-1720, 1992. 